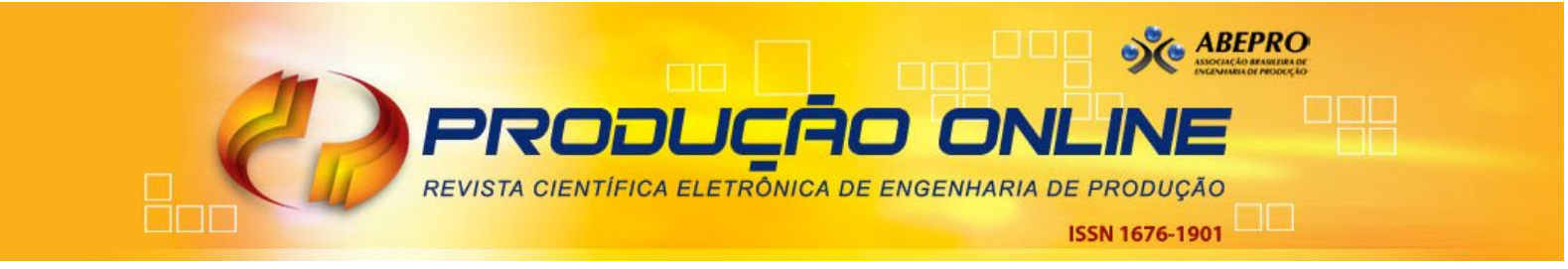

\title{
GESTÃO DE RISCOS EM CADEIA DE SUPRIMENTOS: APLICAÇÃO EM UMA DISTRIBUIDORA DE GÁS CANALIZADO
}

\section{SUPPLY CHAIN RISK MANAGEMENT: APPLICATION IN A CHANNELED GAS DISTRIBUIDOR}

\author{
Ana Camila Rodrigues de Oliveira* E-mail: camilarodrigues091@gmail.com \\ Liane Márcia Freitas e Silva* E-mail: lianemarcia@hotmail.com \\ Maria Silene Alexandre Leite* E-mail: mariasileneleite@hotmail.com \\ *Universidade Federal da Paraíba (UFPB), João Pessoa, PB
}

\begin{abstract}
Resumo: O objetivo deste artigo é identificar, avaliar e priorizar os riscos existentes em uma empresa pertencente a uma cadeia de suprimentos de gás natural canalizado no Estado da Paraíba. A relevância deste trabalho relaciona-se ao fato de que as interrupções em cadeias de suprimentos podem gerar perdas financeiras, elevação dos custos, redução da qualidade do produto e/ou serviço oferecido e perda de reputação. Para alcançar o objetivo proposto, utilizou-se um instrumento de pesquisa construído a partir de uma ampla revisão da literatura. Como resultado, observou-se que o risco de preço é prioritário, uma vez que possui alta probabilidade de ocorrência e pode impactar de forma severa na organização. Tal risco ocorre devido às variações no preço das matérias-primas e às variações no preço do produto final ao cliente. Por fim, a pesquisa sugere estratégias de mitigação para os riscos críticos com a finalidade de assegurar a continuidade dos negócios. No entanto, os riscos que não foram considerados como prioritários não devem ser ignorados, uma vez que novos riscos surgem ao longo do tempo e os riscos existentes podem evoluir.
\end{abstract}

Palavras-chave: Cadeia de suprimentos. Gestão de Riscos. Identificação dos riscos. Avaliação dos riscos. Priorização dos riscos.

\begin{abstract}
The objective of this article is to identify, evaluate and prioritize the existing risks in a company belonging to a piped natural gas supply chain in the State of Paraíba. The relevance of this work is related to the fact that interruptions in supply chains can generate financial losses, increase costs, reduce the quality of the product and / or service offered and loss of reputation. As a result, it was observed that price risk is a priority, since it has a high probability of occurrence and can have a severe impact on the organization. Such risk occurs due to variations in the price of raw materials and variations in the price of the final product to the customer. Finally, the research suggests mitigation strategies for critical risks in order to ensure business continuity. However, risks that were not considered as priorities should not be ignored, as new risks arise over time and existing risks can evolve.
\end{abstract}

Keywords: Supply chain. Risk management. Risk Identification. Risk assessment. Prioritization of risks.

\section{INTRODUÇÃO}

$\mathrm{Na}$ busca por controlar e mitigar os efeitos negativos causados por eventos de risco, muitos modelos têm sido desenvolvidos para a gestão de riscos em cadeias de suprimentos. Segundo Tang e Musa (2011), a gestão de riscos em cadeia de suprimentos (Supply Chain Risk Management - SCRM) se dá por meio da 
coordenação ou colaboração entre os membros da cadeia de suprimentos de modo a garantir o lucro, a capacidade e a continuidade de suas operações.

A gestão de riscos em cadeias de suprimentos é uma área relativamente nova de pesquisa que tem causado interesse nos pesquisadores, uma vez que pode auxiliar a melhorar o desempenho da cadeia como um todo (FAZLI; MAVI; VOSOOGHIDIZAJI, 2015). Sua principal finalidade é proteger os negócios de eventos adversos (COLICCHIA; STROZZI, 2012). Em outras palavras, a SCRM busca reduzir a frequência e/ou o impacto dos riscos na cadeia de suprimentos.

Esse campo de estudo tem como objetivo desenvolver abordagens para identificar, avaliar e analisar as áreas de vulnerabilidade e de risco nas cadeias de suprimentos (FAZLI; MAVI; VOSOOGHIDIZAJI, 2015). Para alcançar tal objetivo, os modelos de gestão de riscos dividem-se em quatro etapas basicamente: identificação do risco, avaliação do risco, mitigação do risco e monitoramento do risco (HO et al., 2015; BLOME; SCHOENHERR, 2011; TUNCEL; ALPAN, 2010; WAGNER; BODE, 2009; HALLIKAS et al., 2004).

No entanto, a implementação da gestão de riscos é uma tarefa extremamente crítica e que exige conhecimento das funções de negócios, tendências de mercado e situação financeira e de infraestrutura da organização, bem como de toda a cadeia de suprimentos (SINGHAL; AGARWAL; MITTAL, 2011). Além disso, as organizações que compõem a cadeia de suprimentos são dinâmicas, dispersas e extremamente interligadas e, consequentemente, o desempenho de uma empresa afeta o desempenho da outra. Como resultado, o gerenciamento dos riscos torna-se cada vez mais complexo (COLICCHIA; DALLARI; MELACINI, 2010; ADHITYA; SRINIVASAN; KARIMI, 2007).

Apesar da existência de vários estudos a respeito da gestão de riscos em cadeias de suprimentos, Ho et al. (2015) argumentam que uma parte significativa desses estudos é de natureza teórica, a exemplo das pesquisas feitas por Rao e Goldsby (2009), Tang e Musa (2011), Sodhi, Son e Tang (2012), Colicchia e Strozzi (2012), Ghadge, Dani e Kalawsky (2012), Heckman, Comes e Nickel (2015) e Qazi, Quigley e Dickson (2015). Além disso, Ho et al. (2015) enfatizam que a maior parte dos estudos em gestão de riscos são realizados nas cadeias da indústria automobilística, da indústria de eletrônicos e da indústria aeroespacial. 
Sendo assim, a indústria de gás natural foi selecionada para a pesquisa por seu papel estratégico como fonte de energia para o mundo e também pela escassez de estudos empíricos nesse setor específico. Vale salientar que uma interrupção no fornecimento de gás natural para as indústrias que a utilizam em seu processo de produção pode gerar um impacto significativo em termos financeiros e de reputação para essas organizações.

Nesse contexto, este artigo tem como objetivo identificar, avaliar e priorizar os riscos existentes em uma empresa distribuidora de gás canalizado por meio de uma classificação de riscos construída a partir de uma ampla revisão da literatura, que resultou em 25 tipos de riscos. Em seguida, os riscos foram avaliados (em termos de probabilidade de ocorrência e severidade) e priorizados (com o auxílio do diagrama de risco).

A estrutura desse artigo segue a ordem descrita: incialmente é feita uma explanação sobre a gestão de riscos na cadeia de suprimentos. Em seguida, o método de pesquisa é descrito. Na sequência, os resultados são apresentados e discutidos e, por fim, as considerações finais são expostas.

\section{GESTÃO DE RISCOS EM CADEIAS DE SUPRIMENTOS}

Os riscos podem surgir a partir de falhas e incertezas nos processos empresariais, da integração com os outros membros da cadeia ou por fatores do ambiente externo, que podem influenciar nos resultados obtidos pela cadeia (GAONKAR; VISWANADHAM, 2007).

Waters (2007) afirma que o risco ocorre porque existe alguma incerteza sobre o futuro. Corroborando com essa opinião, Manuj e Mentzer (2008) argumentam que o risco é o resultado esperado de um evento incerto, ou seja, eventos incertos levam à existência dos riscos. Sendo assim, o risco pode ser compreendido como função do nível de incerteza e do impacto de um evento adverso (SINHA; WHITMAN; MALZAHN, 2004).

Como resultado, a ocorrência de diferentes eventos de riscos em uma determinada organização pode gerar diversas consequências, tais como: perda de vendas, aumento dos custos, perdas financeiras, redução da qualidade do produto, perdas sociais, ameaça à vida e à segurança dos clientes, imagem corporativa 
negativa e atrasos na entrega dos clientes (CERYNO; SCAVARDA; KLINGEBIEL, 2014).

Nesse contexto, buscando proteger os negócios de eventos adversos, implementa-se a gestão de riscos, que tem como objetivo principal reduzir a frequência e/ou o impacto dos riscos nas cadeias de suprimentos. O processo de gestão de riscos, por sua vez, divide-se em quatro etapas: identificação dos riscos, avaliação dos riscos, mitigação dos riscos e monitoramento dos riscos.

A identificação do risco é o primeiro passo para entender as fontes de riscos (tanto internas quanto externas) que as cadeias de suprimentos estão submetidas (HO et al., 2015). No entanto, nem todos os riscos são fáceis de identificar e, portanto, deve-se selecionar um método estruturado para identificação do risco, pois uma má identificação do risco leva a uma avaliação falha do risco que, por sua vez, implica em selecionar uma estratégia de mitigação do risco inadequada, ou seja, a etapa de identificação dos riscos influencia diretamente nas próximas fases do processo de gestão de riscos em cadeias de suprimentos.

Nesse contexto, o Quadro 1 apresenta as diferentes abordagens que podem ser utilizadas para identificar os riscos.

Ainda sobre a identificação do risco, Shi (2004) argumenta que para identificar os riscos de forma eficaz, uma classificação de riscos é bastante útil, uma vez que permitirá que uma determinada organização reconheça os riscos que está submetida. Corroborando com essa realidade, Aqlan (2016) reconhece que é fundamental compreender as diferentes categorias de riscos existentes e, consequentemente, os eventos e condições que originam esses riscos.

Nesse contexto, os estudos empíricos em gestão de riscos abrangem poucos tipos de riscos ou fatores de riscos, como aqueles realizados por Gaudenzi e Borghesi (2006), Wu, Blackhurst e Chidambaram (2006), Schoenherr, Tummala e Harrison (2008), Funo et al. (2011) e Radivojević e Gajović (2014).

Buscando identificar os riscos de forma mais aprofundada, esta pesquisa envolve 25 tipos de riscos, conforme Quadro 2. Sendo assim, torna-se possível enxergar os riscos existentes em uma determinada organização de forma mais completa. 
Quadro 1 - Abordagens para realizar a identificação do risco

\begin{tabular}{|c|c|}
\hline $\begin{array}{l}\text { Abordagens utilizadas } \\
\text { para identificar o risco }\end{array}$ & Descrição \\
\hline $\begin{array}{l}\text { Mapeamento da cadeia de } \\
\text { suprimentos }\end{array}$ & $\begin{array}{l}\text { A cadeia de suprimentos e seus fluxos de bens, informações e } \\
\text { financeiro são representados visualmente, desde fornecedores até os } \\
\text { clientes. Uma vez que todos os detalhes da cadeia tenham sido } \\
\text { mapeados, os riscos potenciais podem ser identificados. }\end{array}$ \\
\hline Checklist & $\begin{array}{l}\text { Listas de verificação são formas para registrar quantas vezes uma falha } \\
\text { foi atribuída a um evento específico. Poderia ser utilizada, por exemplo, } \\
\text { para registrar atrasos nas entregas de fornecedores, que podem servir } \\
\text { como informações para avaliar sua confiabilidade. }\end{array}$ \\
\hline Análise da árvore de falhas & $\begin{array}{l}\text { Examina todos os possíveis eventos que antecederam o evento crítico } \\
\text { e um diagrama gráfico mostra como o sistema pode falhar. }\end{array}$ \\
\hline $\begin{array}{l}\text { Análise da árvore de } \\
\quad \text { eventos }\end{array}$ & $\begin{array}{l}\text { É um diagrama lógico que foca nos eventos que podem ocorrer após a } \\
\text { existência de um evento crítico. Além disso, identifica e quantifica os } \\
\text { possíveis resultados seguintes. }\end{array}$ \\
\hline $\begin{array}{l}\text { Análise do tipo e efeito da } \\
\text { falha (FMEA) }\end{array}$ & $\begin{array}{l}\text { É uma ferramenta para identificar, analisar e priorizar as falhas } \\
\text { potencias. É uma ferramenta proativa, uma vez que é utilizada para } \\
\text { prever e eliminar modos de falha potenciais em um sistema antes } \\
\text { dessas falhas ocorrerem. }\end{array}$ \\
\hline $\begin{array}{l}\text { Análise dos Efeitos e } \\
\text { criticidades dos Modos de } \\
\text { Falha (FMECA) }\end{array}$ & $\begin{array}{l}\text { FMECA é uma extensão do FMEA. As entradas nessa abordagem são: } \\
\text { planos, diagramas, probabilidades e frequências baseadas no } \\
\text { conhecimento histórico. } \\
\text { Como resultado, FMECA fornece uma lista dos riscos mais críticos, bem } \\
\text { como algumas ações de mitigação. }\end{array}$ \\
\hline Diagrama de causa e efeito & $\begin{array}{l}\text { Envolve a análise de brainstorming e exploração de todas as relações } \\
\text { entre as possíveis causas e eventos de falha. Uma vez que uma falha } \\
\text { é identificada, esses diagramas podem ser utilizados para descobrir a } \\
\text { verdadeira causa do incidente. }\end{array}$ \\
\hline HazOP & $\begin{array}{l}\text { Um estudo HazOP é uma análise estruturada de um sistema, processo } \\
\text { ou operação realizada por uma equipe multidisciplinar. } \\
\text { O estudo é feito usando um conjunto de palavras-guia em combinação } \\
\text { com os parâmetros do sistema para encontrar desvios. }\end{array}$ \\
\hline
\end{tabular}

Fonte: Lavastre, Gunasekaran e Spalanzani (2014); Colicchia e Strozzi (2012); Tummala e Schoenherr (2011); Tuncel e Alpan (2010); Ahmed, Kayis e Amornsawadwatana (2007); Norrman e Jansson (2004) 
Quadro 2 - Tipos de riscos

\begin{tabular}{|c|c|}
\hline TIPO DE RISCO & AUTORES \\
\hline Risco ambiental & $\begin{array}{l}\text { Jüttner, Peck e Christopher (2003), Zsidisin et al. (2000), Hallikas et al. } \\
\text { (2004), Christopher e Peck (2004), Zsidisin (2003), Juttner (2005), } \\
\text { Matook, Lasch e Tamaschke (2009), Rao e Goldsby (2009), Samvedi, } \\
\text { Jain e Chan (2013) }\end{array}$ \\
\hline $\begin{array}{l}\text { Risco } \\
\text { competitivo/estratégico }\end{array}$ & Harland, Brenchley e Walker (2003), Manuj e Mentzer (2008) \\
\hline $\begin{array}{l}\text { Risco de } \\
\text { processo/controle }\end{array}$ & $\begin{array}{l}\text { Tang e Tomlin (2008), Matook, Lasch e Tamaschke, (2009), Schoenherr } \\
\text { (2011), Christopher, Mena, Khan e Yurt (2011) }\end{array}$ \\
\hline Risco de demanda & $\begin{array}{l}\text { Hallikas et al. (2004), Juttner (2005), Manuj e Mentzer (2008), Tang e } \\
\text { Tomlin (2008), Tummala e Schoenherr (2011), Ghadge, Dani e Kalawsky } \\
\text { (2012) }\end{array}$ \\
\hline Risco de mercado & Trkman e McCormack (2009) \\
\hline $\begin{array}{l}\text { Risco } \\
\text { capacidade/recurso }\end{array}$ & $\begin{array}{l}\text { Hallikas et al. (2004), Manuj e Mentzer (2008b), Matook, Lasch e } \\
\text { Tamaschke, (2009), Tummala e Schoenherr (2011) }\end{array}$ \\
\hline Risco de segurança & Manuj e Mentzer (2008b), Ojha e Gokhale (2009) \\
\hline Risco de fornecimento & $\begin{array}{l}\text { Harland, Brenchley e Walker (2003), Manuj e Mentzer (2008b), Tang e } \\
\text { Tomlin (2008), Christopher, Mena, Khan e Yurt (2011), Tummala e } \\
\text { Schoenherr (2011), Ghadge, Dani e Kalawsky (2012), Punniyamoorthy, } \\
\text { Thamaraiselvan, Manikandan (2013), Ho et al. (2015) }\end{array}$ \\
\hline Riscos logísticos & Punniyamoorthy, Thamaraiselvan, Manikandan (2013) \\
\hline $\begin{array}{l}\text { Risco } \\
\text { econômico/financeiro }\end{array}$ & $\begin{array}{l}\text { Zsidisin (2003), Harland, Brenchley e Walker (2003), Manuj e Mentzer } \\
\text { (2008), Matook, Lasch e Tamaschke, (2009), Funo et al. (2011) }\end{array}$ \\
\hline $\begin{array}{l}\text { Risco } \\
\text { industrial/operacional/de } \\
\text { manufatura }\end{array}$ & $\begin{array}{l}\text { Harland, Brenchley e Walker (2003), Han e Chen (2007), Manuj e } \\
\text { Mentzer (2008), Ojha e Gokhale (2009), Matook, Lasch e Tamaschke, } \\
\text { (2009), Lockamy III e McCormack (2010), Tummala e Schoenherr } \\
\text { (2011), Punniyamoorthy, Thamaraiselvan, Manikandan (2013), Ho et al. } \\
\text { (2015) }\end{array}$ \\
\hline Risco político & $\begin{array}{l}\text { Juttner. (2005), Tang e Tomlin (2008), Manuj e Mentzer (2008b), Funo } \\
\text { et al. (2011) }\end{array}$ \\
\hline Risco tecnológico & $\begin{array}{l}\text { Zsidisin et al. (2000), Chopra e Sodhi (2004), Trkman e McCormack } \\
\text { (2009), Matook, Lasch e Tamaschke, (2009), Funo et al. (2011) }\end{array}$ \\
\hline $\begin{array}{l}\text { Risco de propriedade } \\
\text { intelectual }\end{array}$ & Sodhi e Tang (2009) \\
\hline Risco de preço & Matook, Lasch e Tamaschke, (2009) \\
\hline Risco de cliente & Harland, Brenchley e Walker (2003) \\
\hline $\begin{array}{l}\text { Risco de depreciação de } \\
\text { ativos }\end{array}$ & Harland, Brenchley e Walker (2003), Wang e Yang (2009) \\
\hline Risco de reputação & Harland, Brenchley e Walker (2003) \\
\hline Risco fiscal & Harland, Brenchley e Walker (2003) \\
\hline Risco legal & Harland, Brenchley e Walker (2003) e Zsidisin (2003) \\
\hline Risco de informação & $\begin{array}{l}\text { Cavinato (2004), Wang e Yang (2009), } \\
\text { Thamaraiselvan, Manikandan (2013) }\end{array}$ \\
\hline Risco comportamental & Tang e Tomlin (2008) \\
\hline Risco de estoque & Diabat, Govindan e Panicker (2012) \\
\hline Risco Cultural & Rangel, Oliveira e leite (2014) \\
\hline Risco de Design & Ghadge et al. (2013), Aqlan e Lam (2015) \\
\hline
\end{tabular}

Fonte: Elaboração própria (2018) 
A segunda etapa do processo de gestão de riscos é a avaliação do risco. Nesta etapa, duas dimensões precisam ser consideradas: frequência/probabilidade e severidade/consequência (AQLAN; LAM, 2015; GARVEY; CARNOVALE; YENIYURT, 2015).

A terceira etapa do processo de gestão de riscos compreende 0 estabelecimento de estratégias de mitigação do risco, que são ações com a finalidade de diminuir as incertezas identificadas a partir de várias fontes de riscos rastreadas inicialmente (CERYNO; SCAVARDA; KLINGEBIEL, 2014). Para Aqlam e Lam (2015), as estratégicas de mitigação dos riscos em cadeias de suprimentos são:

a) Evitar o risco - O risco pode ser evitado por meio da eliminação de suas causas raízes e/ou consequências. É uma estratégia adequada quando existe alta probabilidade de ocorrência do risco e alto impacto do risco, sendo assim, estes riscos são críticos e podem interromper as operações da cadeia;

b) Reduzir o risco - Significa que o risco pode ser reduzido, mas não eliminado. Essa estratégia é adequada quando o risco tem alta probabilidade de ocorrência e baixo impacto, sendo assim, estes riscos são aqueles operacionais e do dia-a-dia. Algumas técnicas para redução do risco são: redundância, maior quantidade de testes de qualidade, ferramentas melhores e outras;

c) Transferir o risco - O risco é transferido para outra parte. É adequado quando o risco tem baixa probabilidade de ocorrência, no entanto, possui alto impacto, tais como: desastres naturais e ataques terroristas. Métodos comuns incluem: seguro e contratos;

d) Aceitar o risco - É uma estratégia adequada quando existe baixa probabilidade de ocorrência do risco e alto impacto, uma vez que o custo de evitar/reduzir/transferir o risco é muito alto quando comparado ao impacto esperado. Os planos de contingência devem ser desenvolvidos para lidar com esse tipo de risco;

e) Ignorar o risco - Os riscos são ignorados e não são identificados nem estudados. As organizações assumem os riscos quando estes possuem baixa probabilidade de ocorrência e baixo impacto, no entanto, ignorar um risco pode representar um risco;

f) Explorar o risco - É o oposto de evitar. Considera-se que o impacto do risco é positivo. 
Para Kern et al. (2012), as atividades de mitigação só funcionam bem quando a avaliação dos riscos é realizada de forma minuciosa porque todas as atividades de mitigação dependem fortemente do entendimento da etapa anterior (avaliação do risco). Dessa forma, os dados coletados nas etapas anteriores do processo de gestão de riscos são utilizados para estabelecer as medidas adequadas. Isso inclui estratégias clássicas de mitigação (antes do evento de risco) e planos de contingência (após a ocorrência do evento de risco), como apontado por Kern et al. (2012).

A última etapa do processo de gestão de riscos é o monitoramento do risco. É nesta etapa que o sistema deve ser monitorado continuamente para detectar os riscos no momento adequado (TUNCEL; ALPAN, 2010). Entretanto, Ho et al. (2015) alertam que esta etapa do processo tem sido pouco estudada, quando comparada com as outras etapas.

Considerando o exposto, a próxima seção trata dos procedimentos metodológicos utilizados para o desenvolvimento desta pesquisa.

\section{METODOLOGIA}

A pesquisa foi realizada no Brasil entre julho e novembro do ano de 2016 e foi dividida em três grandes etapas distintas, conforme ilustra Figura 1.

Figura 1 - Etapas da pesquisa
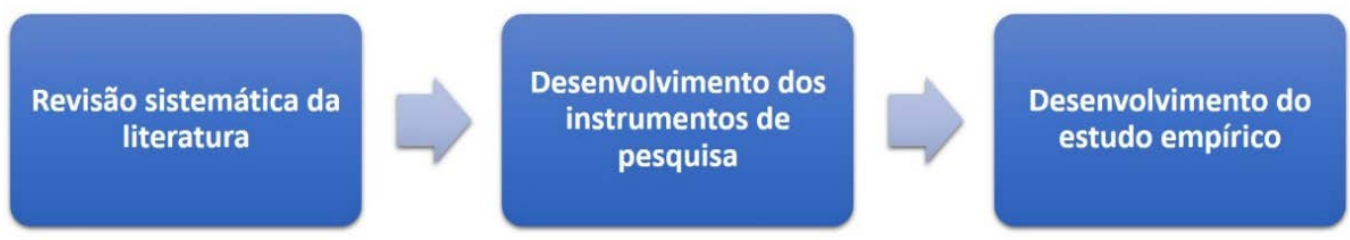

Fonte: Elaboração própria (2018)

\subsection{Revisão Sistemática da Literatura}

Uma revisão sistemática da literatura foi realizada para compreender os diferentes tipos de riscos existentes. Para tal, adotou-se o procedimento descrito por Lacerda, Ensslin e Ensslin (2012). As bases de dados utilizadas para a composição 
do portfólio de artigos incluíram: Emerald, Science Direct, Taylor and Francis e Web of Science.

As palavras-chave utilizadas para conduzir a revisão sistemática sobre a gestão de riscos em cadeias de suprimentos foram: "risk", "supply chain risk management" "risk assessment", "risk evaluation", "risk identification", "resilience", "risk analysis", "risk management", "supply chain", "vulnerability" e "sources of risk". Além disso, o período de tempo definido para a pesquisa dos artigos foi entre 2005 e 2015 . No entanto, estudos realizados em períodos anteriores e que são considerados relevantes também foram incorporados à pesquisa. Em seguida, iniciou-se o processo de filtragem de artigos com a finalidade de obter o material necessário para a revisão sistemática, conforme apresenta a Figura 2.

Figura 2 - Procedimento adotado para seleção do portfólio de artigos

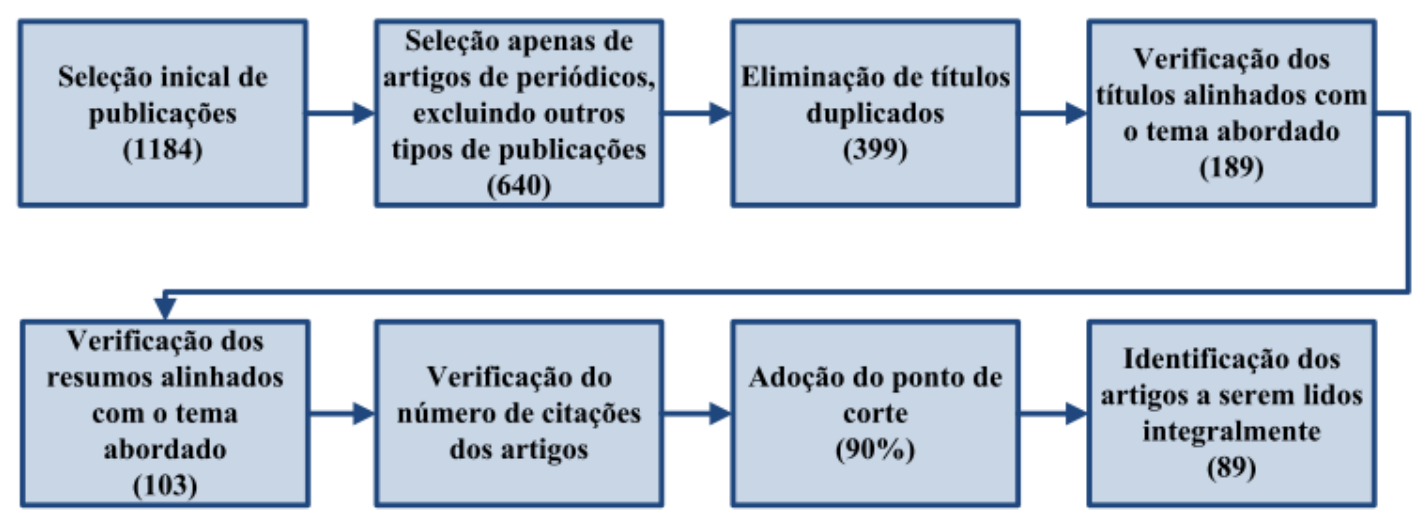

Fonte: Elaboração própria, com base em Lacerda, Ensslin e Ensslin (2012)

Verifica-se assim que a seleção inicial de publicações sobre o tema abordado na pesquisa consistiu em 1.184 publicações, conforme demonstra Figura 2. Após realizar todos os filtros, foi possível chegar a um portfólio de 89 artigos, que foram lidos integralmente.

\subsection{Desenvolvimento do Instrumento de Pesquisa}

Buscando alcançar o objetivo proposto neste artigo, um instrumento de pesquisa foi elaborado, englobando 25 tipos de riscos com base em classificações de riscos encontradas na literatura. Esses diferentes tipos de riscos foram fragmentados em fatores de riscos, com a finalidade de obter um questionário mais robusto e de fácil 
entendimento pelos respondentes selecionados, conforme Quadro 3. Esses fatores de riscos foram estruturados com base nos artigos dos seguintes autores: Harland, Brenchley e Walker (2003), Gaudenzi e Borghesi (2006), Blackhurst, Scheibe e Johnson (2008), Tang e Tomlin (2008), Trkman e McCormack (2009), Matook, Lasch e Tamaschke, (2009), Punniyamoorthy, Thamaraiselvan e Manikandan (2013) e Ho et al. (2015).

Salienta-se que o questionário apresentado é um recorte e, dessa forma, todos os riscos investigados não estão presentes no quadro citado, que foram: risco ambiental, risco de segurança, riscos político/social, risco de propriedade intelectual, risco de preço, risco de reputação, risco comportamental, risco legal, risco estratégico, risco de mercado, risco de processo/controle, risco de demanda, risco de capacidade, risco operacional, risco de fornecimento, risco logístico, risco de estoque, risco tecnológico, risco de informação, risco de cliente, risco cultural, risco de design, risco econômico/financeiro, risco fiscal e depreciação de ativos. 
Quadro 3 - Recorte do questionário utilizado para identificação e avaliação dos riscos

\begin{tabular}{|c|c|c|c|c|c|c|c|c|}
\hline \multirow{2}{*}{$\begin{array}{l}\text { TIPOS DE } \\
\text { RISCOS }\end{array}$} & \multirow{2}{*}{ FATORES DE RISCOS } & \multicolumn{3}{|c|}{ Existe? } & \multicolumn{3}{|c|}{$\begin{array}{c}\text { Probabilidade de } \\
\text { ocorrência }\end{array}$} & \multirow{2}{*}{$\begin{array}{c}\text { Qual o impacto } \\
\text { do risco? }\end{array}$} \\
\hline & & SIM & NÃO & $\begin{array}{l}\text { NÃO } \\
\text { SEI }\end{array}$ & Baixa & Média & Alta & \\
\hline \multirow{5}{*}{ ESTRATÉGICO } & $\begin{array}{l}\text { 1. O posicionamento estratégico da empresa já foi afetado } \\
\text { negativamente pela ação dos concorrentes em diferenciar seus } \\
\text { produtos? }\end{array}$ & & & & & & & \multirow{5}{*}{$\begin{array}{l}\text { A) Severo } \\
\text { B) Alto } \\
\text { C) Médio } \\
\text { D)Pequeno } \\
\text { E) Nenhum }\end{array}$} \\
\hline & $\begin{array}{l}\text { 2. O posicionamento estratégico da empresa já foi afetado } \\
\text { negativamente pela não capacidade de diferenciação dos produtos } \\
\text { da empresa? }\end{array}$ & & & & & & & \\
\hline & $\begin{array}{l}\text { 3. O posicionamento estratégico da empresa já foi afetado } \\
\text { negativamente pelo não conhecimento das habilidades dos } \\
\text { concorrentes? }\end{array}$ & & & & & & & \\
\hline & $\begin{array}{l}\text { 4. O posicionamento estratégico da empresa já foi afetado } \\
\text { negativamente pelas mudanças de preços praticadas pelos } \\
\text { concorrentes? }\end{array}$ & & & & & & & \\
\hline & $\begin{array}{l}\text { 5. O posicionamento estratégico da empresa já foi afetado } \\
\text { negativamente pela não capacidade de diferenciar o produto da } \\
\text { empresa dos produtos concorrentes? }\end{array}$ & & & & & & & \\
\hline \multirow{7}{*}{ OPERACIONAL } & $\begin{array}{l}\text { 1. A empresa já interrompeu suas operações devido a falhas na } \\
\text { manutenção? }\end{array}$ & & & & & & & \multirow{7}{*}{$\begin{array}{l}\text { A) Severo } \\
\text { B) Alto } \\
\text { C) Médio } \\
\text { D)Pequeno } \\
\text { E)Nenhum }\end{array}$} \\
\hline & $\begin{array}{l}\text { 2. A empresa já interrompeu suas operações devido a falhas } \\
\text { humanas na operabilidade diária? }\end{array}$ & & & & & & & \\
\hline & $\begin{array}{l}\text { 3. A empresa já interrompeu suas operações devido à falhas na } \\
\text { programação das atividades? }\end{array}$ & & & & & & & \\
\hline & $\begin{array}{l}\text { 4. A empresa já enfrentou problemas por não entregar seus produtos } \\
\text { com a qualidade requerida? }\end{array}$ & & & & & & & \\
\hline & $\begin{array}{l}\text { 5. A empresa já enfrentou problemas por não entregar seus produtos } \\
\text { no tempo esperado pelo cliente? }\end{array}$ & & & & & & & \\
\hline & $\begin{array}{l}\text { 6. A empresa já enfrentou problemas por não entregar seus produtos } \\
\text { na quantidade esperada pelo cliente? }\end{array}$ & & & & & & & \\
\hline & $\begin{array}{l}\text { 7. A empresa já enfrentou problemas por não seguir os } \\
\text { procedimentos padrões para execução das operações do processo? }\end{array}$ & & & & & & & \\
\hline
\end{tabular}

Fonte: Elaboração Própria (2018) 
Para mensurar a probabilidade de ocorrência, um campo foi incorporado no questionário para que o respondente informe essa probabilidade para aqueles riscos que foram identificados na organização, conforme Quadro 4.

\begin{tabular}{|c|c|c|c|}
\hline \multicolumn{3}{|c|}{ Probabilidade de ocorrência } & \multirow{2}{*}{$\begin{array}{l}\text { Qual o impacto do } \\
\text { risco? }\end{array}$} \\
\hline Mínima & Média & Máxima & \\
\hline & & & $\begin{array}{l}\text { A) Severo } \\
\text { B) Alto } \\
\text { C) Médio } \\
\text { D)Pequeno } \\
\text { E)Nenhum }\end{array}$ \\
\hline
\end{tabular}

Fonte: Elaboração própria (2018)

Essa probabilidade pode ser considerada mínima, média ou máxima, de acordo com a opinião dos especialistas. Sobre a utilização da opinião dos especialistas, Radivojević e Gajović (2014) explicam que os riscos geralmente são avaliados com base na intuição, experiência, conhecimento ou consequências.

Corroborando com os autores mencionados, também utilizaram a opinião de especialistas para avaliar os riscos nas cadeias de suprimentos: Aqlan (2016), Aqlan e Lam (2015), Fazli, Mavi e Vosooghidizaji (2015), Bradley (2014), Lavastre, Gunasekaran e Spalanzani (2014), Wu et al. (2013), Kern et al. (2012), Lockamy III e McCormack (2012), Vilko e Hallikas (2012), Blome e Schoenherr (2011), Olson e Wu (2011), Tummala e Schoenherr (2011), Thun e Hoenig (2011), Manuj, Mentzer e Bowers (2009), Wu, Backhurst e Chidambaram (2006).

Já o impacto do risco foi mensurado de forma qualitativa, conforme Quadro 4. Os gestores responderam se um determinado risco era capaz de gerar um impacto severo, alto, médio, pequeno ou nenhum para os negócios da organização.

Na sequência, o instrumento de pesquisa foi testado e calibrado. Tal teste foi aplicado em três organizações localizadas no Estado da Paraíba pertencentes aos setores de alimentos e cerâmico com a finalidade de identificar os riscos e avaliá-los em termos de probabilidade de ocorrência e impacto.

\subsection{Desenvolvimento do Estudo Empírico}

Em seguida, uma empresa foi selecionada para o estudo. Tal empresa dedica- 
se à exploração exclusiva da atividade de distribuição de gás canalizado no Estado da Paraíba e essa matriz energética é de extrema importância para o funcionamento das empresas locais.

Os riscos existentes nas organizações foram identificados e avaliados com 0 auxílio do instrumento de pesquisa elaborado, ilustrado no Quadro 3. Os respondentes pertencem ao nível gerencial. No total, seis (6) gerentes foram envolvidos na pesquisa, conforme Quadro 5. Acredita-se que melhores resultados podem ser obtidos quando os questionamentos são realizados para aqueles que são especialistas em um determinado assunto e, por isso, os questionários foram divididos de acordo com cada gerência. Como exemplo, pode-se citar os riscos econômicos/financeiros, pois os indivíduos pertencentes à gerência de finanças possuem maior habilidade para responder questionamentos sobre esse tema especifico.

Dessa forma, considerou-se que se um fator de risco ligado a uma categoria de risco for identificado, então o risco existe na empresa investigada. O preenchimento dos questionários foi efetuado pelo pesquisador, após a leitura da pergunta com suas respectivas opções de resposta. Nesse sentido, a resposta verbal dada pelo entrevistado foi preenchida pelo pesquisador.

Quadro 5 - Perfil dos entrevistados

\begin{tabular}{|c|c|c|c|c|c|}
\hline Empresa & Data & $\begin{array}{l}\text { Participantes da } \\
\text { empresa focal }\end{array}$ & $\begin{array}{c}\text { Tempo de } \\
\text { atuação } \\
\text { na } \\
\text { empresa }\end{array}$ & Formação & $\begin{array}{l}\text { Duração } \\
\text { da vista }\end{array}$ \\
\hline \multirow{6}{*}{ 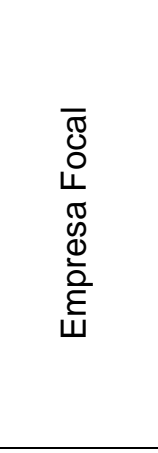 } & \multirow{4}{*}{$\begin{array}{l}18 \text { de julho } \\
\text { de } 2016\end{array}$} & $\begin{array}{c}\text { Gerente Industrial e } \\
\text { Automotivo }\end{array}$ & 8 anos & $\begin{array}{c}\text { Engenheiro } \\
\text { mecânico }\end{array}$ & \multirow{4}{*}{3 horas } \\
\hline & & $\begin{array}{l}\text { Gerente de } \\
\text { Suprimentos }\end{array}$ & 8 anos & Contador & \\
\hline & & $\begin{array}{c}\text { Gerente de Tecnologia } \\
\text { da Informação }\end{array}$ & 4 anos & $\begin{array}{l}\text { Ciências da } \\
\text { Computação }\end{array}$ & \\
\hline & & Gerente de Marketing & 5 anos & Administrador & \\
\hline & $\begin{array}{c}21 \text { de julho } \\
\text { de } 2016 \\
\end{array}$ & $\begin{array}{c}\text { Diretor-técnico } \\
\text { comercial }\end{array}$ & 10 anos & $\begin{array}{c}\text { Engenheiro } \\
\text { civil }\end{array}$ & $\begin{array}{c}1 \text { hora e } 20 \\
\text { minutos }\end{array}$ \\
\hline & $\begin{array}{l}28 \text { de julho } \\
\text { de } 2016\end{array}$ & Gerente Financeiro & 9 anos & Administrador & 1 hora \\
\hline
\end{tabular}

Fonte: Elaboração própria (2018)

Dessa forma, os riscos foram identificados e avaliados pelos gestores mencionados no Quadro 5. Em seguida, com os parâmetros de severidade e 
frequência de ocorrência mapeados, tornou-se possível priorizar os riscos mais críticos utilizando matrizes de severidade e probabilidade.

Após a hierarquização dos riscos, tornou-se viável direcionar qual a medida de mitigação dos riscos poderá ser assumida.

A próxima seção apresenta os resultados alcançados nesta pesquisa.

\section{RESULTADOS}

Esta seção apresenta os principais resultados obtidos nesta pesquisa, incluindo a identificação e priorização dos riscos.

\subsection{Caracterização da empresa estudada}

A empresa estudada foi fundada no dia 25 de outubro de 1994 sob a forma de economia mista. O início de suas atividades ocorreu em 1995, quando a empresa passou a se dedicar à exploração exclusiva da atividade de distribuição de gás canalizado no Estado da Paraíba.

A Petrobras é a companhia que explora e transporta o gás natural no Brasil, de forma isolada ou em conjunto com a iniciativa privada (como é o caso do gasoduto Bolívia/Brasil). No que diz respeito à distribuição desse gás natural, o país possui empresas, das quais a maioria conta com a participação da Petrobras no capital acionário, como é o caso da empresa em estudo.

O gás natural é um energético bastante versátil e pode ser utilizado nas mais diversas aplicações, desde a geração de energia elétrica até na produção de calor e vapor. Nesse contexto, a empresa aplica o gás natural nos seguintes segmentos: residencial, comercial, industrial e automotivo. A Figura 3 apresenta cada segmento com a quantidade de estabelecimentos que a empresa fornece o gás natural atualmente.

Destaca-se que, em termos de volume de gás natural consumido, o segmento industrial consome a maior parte desse volume total, destacando a importância desse segmento. Nesse sentido, esta pesquisa identificou e priorizou os riscos presentes em uma empresa distribuidora de gás natural. É importante salientar que tal empresa está 
submetida a diferentes riscos, dentre eles, destaca-se a possibilidade de substituição da matriz energética de seus clientes.

Figura 3 - Segmentos de atuação da empresa

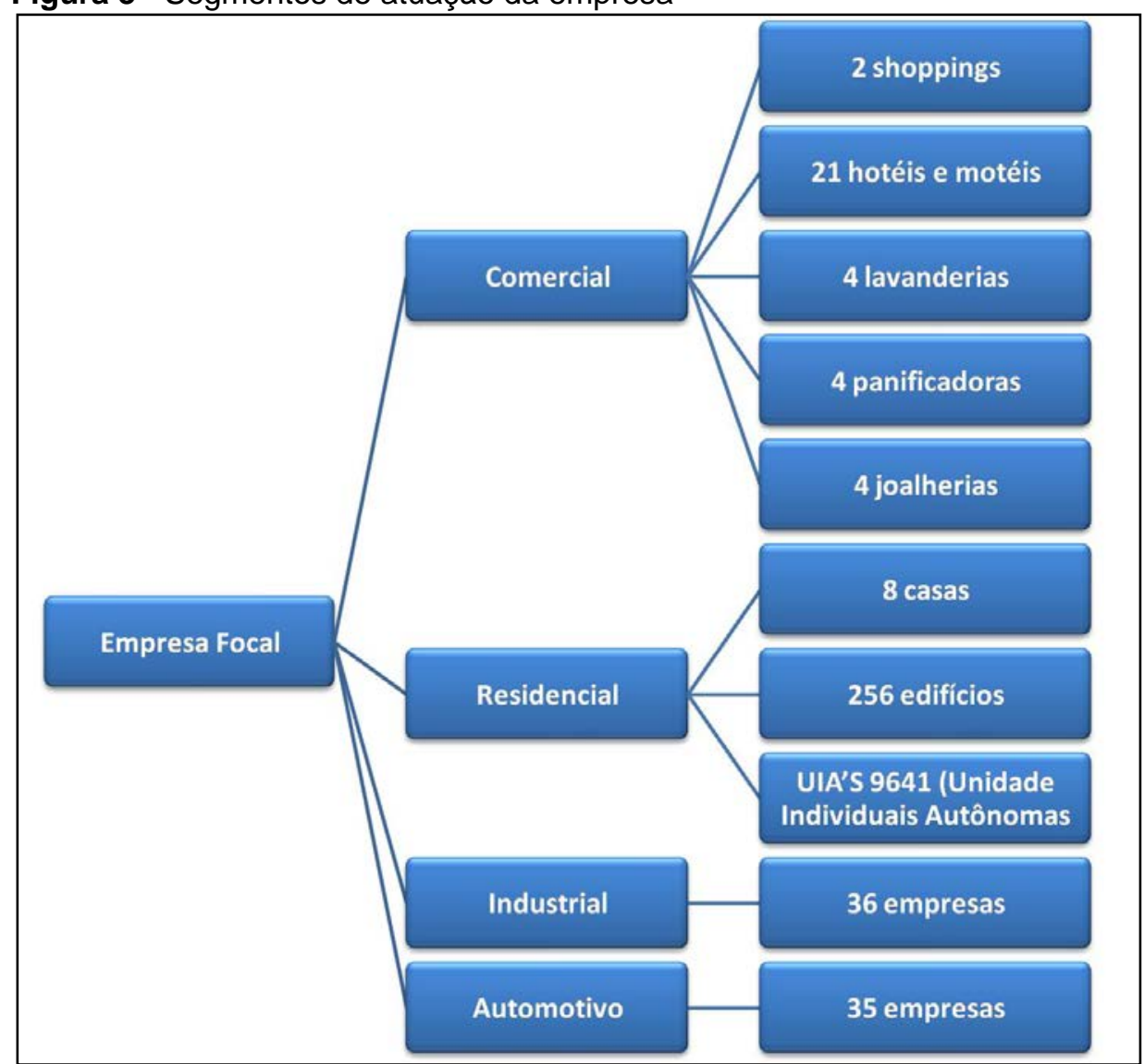

Fonte: Elaboração própria (2017)

\subsection{Identificação dos riscos}

Os riscos identificados na organização objeto de estudo estão expostos no Quadro 6. Foram identificados 13 tipos de riscos dentre todos os listados nos questionários aplicados. Vale salientar que a organização também foi afetada pela crise econômica fortemente, resultando na pior fase da história da empresa em termos de inadimplência e também de menor consumo de gás natural por parte de seus clientes, deixando a empresa exposta aos riscos tanto internos como externos. 
Quadro 6 - Riscos identificados

\begin{tabular}{|c|c|}
\hline Tipo de Risco & Fatores de Riscos \\
\hline Estratégico & Mudanças de preços praticadas pelos concorrentes \\
\hline Processo/Controle & Falta de controle dos processos dos parceiros de negócios \\
\hline \multirow{3}{*}{ Demanda } & Menor consumo pelos clientes \\
\hline & Falta de previsão de demanda \\
\hline & Erros na previsão de demanda \\
\hline Capacidade/Recurso & Falta de redundância de algum recurso \\
\hline Operacional & Não seguir os procedimentos padrões para execução das operações \\
\hline \multirow{3}{*}{ Fornecimento } & Problemas na quantidade de matéria-prima \\
\hline & Problemas na qualidade da matéria-prima \\
\hline & Necessidade de mudança de fornecedores de maneira inesperada \\
\hline \multirow{3}{*}{ Estoque } & Falta de itens em estoque \\
\hline & Perda por obsolescência de insumos estocados \\
\hline & Falhas no planejamento de estoques \\
\hline \multirow{2}{*}{ Informação } & Indisponibilidade no sistema de informação \\
\hline & Atrasos nas informações \\
\hline Cliente & Escolha por produtos similares/substitutos \\
\hline Político & Mudanças no ambiente político nacional \\
\hline Propriedade Intelectual & Limitações financeiras destinados à P\&D \\
\hline \multirow{2}{*}{ Preço } & Variações no preço das matérias primas \\
\hline & Variações no preço do produto final ao cliente \\
\hline \multirow{4}{*}{ Econômicos/Financeiros } & Mudanças na conjuntura econômica do país \\
\hline & Mudanças na conjuntura econômica mundial \\
\hline & A incerteza econômica do país ou mundial \\
\hline & Elevação de parâmetros econômicos como taxas de juros e/ou inflação \\
\hline
\end{tabular}

Fonte: Elaboração própria (2018)

De maneira geral, verificou-se que a situação econômica do país e as decisões políticas são capazes de afetar fortemente a organização, uma vez que a empresa é de economia mista e que o gás natural é uma commodity.

Diante desse contexto, os riscos internos são mais controláveis pela empresa e, para esses, a organização dispõe de registros em seu sistema de informação, enquanto que os riscos externos à empresa possuem poucos registros. Por exemplo, a organização é capaz de informar quantas falhas ocorreram no fornecimento devido à falta de qualidade dos produtos entregues.

\subsection{Probabilidade de ocorrência e Impacto dos Riscos}

O Quadro 7 apresenta a probabilidade de ocorrência e o impacto dos riscos 
identificados na empresa. A probabilidade de ocorrência do risco foi mensurada de forma qualitativa e, dessa forma, os gestores responderam se um determinado risco tinha uma probabilidade de ocorrência baixa, média ou alta. De forma análoga, o impacto foi mensurado de forma qualitativa e, sendo assim, os gestores responderam se um determinado risco era capaz de gerar um impacto severo, alto, médio, pequeno ou nenhum para os negócios da organização.

Quadro 7- Probabilidade e Impacto dos riscos na empresa

\begin{tabular}{|c|c|c|}
\hline Tipo de risco & Probabilidade & Impacto \\
\hline Estratégico & Baixa & Severo \\
\hline Processo/Controle & Baixa & Seveno \\
\hline Demanda & Baixa & Pequeno \\
\hline Capacidade/Recurso & Baixa & Pequeno \\
\hline Operacional & Baixa & Nenhum \\
\hline Fornecimento & Baixa & Pequeno \\
\hline Estoque & Média & Severo \\
\hline Informação & Média & Médio \\
\hline Cliente & Baixa & Alta \\
\hline Político & Baixa & Severo \\
\hline Propriedade Intelectual & Baixa & Médio \\
\hline Preço & Alta & Média \\
\hline Econômicos/Financeiros & & \\
\hline
\end{tabular}

Fonte: Elaboração própria (2018)

Ainda de acordo com o Quadro 7, apenas o risco de preço possui uma alta probabilidade de ocorrência, de acordo com os especialistas. Enquanto que o risco de estoque, de informação e o risco econômico possuem probabilidade de ocorrência média. $\mathrm{E}$ os outros riscos identificados possuem uma baixa probabilidade de ocorrência na organização.

Por outro lado, o risco estratégico, demanda, informação e preço impactam de forma severa nos negócios da empresa. Desse modo, observa-se que para avaliar os riscos de forma correta, torna-se necessário levar em consideração tanto a dimensão probabilidade de ocorrência do risco como a dimensão impacto do risco.

O risco estratégico possui um impacto severo, uma vez que a mudança de preços praticada pelos concorrentes pode provocar uma mudança na utilização da matriz energética, isto é, um determinado cliente pode deixar de utilizar o gás natural para utilizar outra fonte energética, como a biomassa, por exemplo.

O risco de demanda também possui um impacto severo porque caso um determinado cliente reduza seu consumo de gás natural, a empresa sofre quedas em seu faturamento e, dependendo do cliente, essa queda pode ser bastante significativa. 
No momento atual de crise econômica, as empresas reduziram seu volume de produção, o que reduz também a demanda pelo gás natural.

O risco de informação ocorre nos momentos em que há indisponibilidade no sistema de informação da empresa, provocando atrasos em seus procedimentos internos. O impacto foi considerado severo porque esses atrasos podem gerar informações não realistas e perturbar os negócios da empresa.

E o risco de preço é extremamente importante, pois uma variação do preço do gás natural impacta fortemente os negócios da empresa. Isso ocorre porque um aumento no preço dessa matéria-prima vai precisar ser repassado para seus clientes, que a partir disso podem procurar por outras matrizes energéticas mais econômicas.

O risco de propriedade intelectual possui um impacto alto, uma vez que a empresa já enfrentou momentos que precisou limitar os recursos destinados à P\&D.

O risco econômico/financeiro e o risco político possuem impacto médio. O risco de processo/controle, capacidade/recurso, operacional, estoque e cliente impactam pouco nos negócios da organização. Os gestores consideraram que uma parte desses riscos está no âmbito de controle da empresa, como o risco de processo, capacidade, operacional e estoque. No entanto, o risco de cliente, que é caracterizado pela vontade do cliente de escolher um produto para substituir o gás natural não é controlável, mas as ações que a empresa desenvolve para mostrar aos clientes as vantagens na utilização do gás natural reduzem o impacto desse risco.

Por último, o risco de fornecimento não impacta de forma alguma nos negócios da empresa, uma vez que as falhas nos suprimentos de materiais de consumo não são graves.

\subsection{Discussão dos Resultados}

De acordo com as informações obtidas a respeito da probabilidade de ocorrência e do impacto dos riscos, os resultados obtidos podem ser analisados de forma mais profunda.

Com o objetivo de visualizar melhor quais riscos são mais críticos para a organização investigada, um diagrama de risco foi construído, conforme Figura 4. 
Figura 4 - Diagrama de Risco

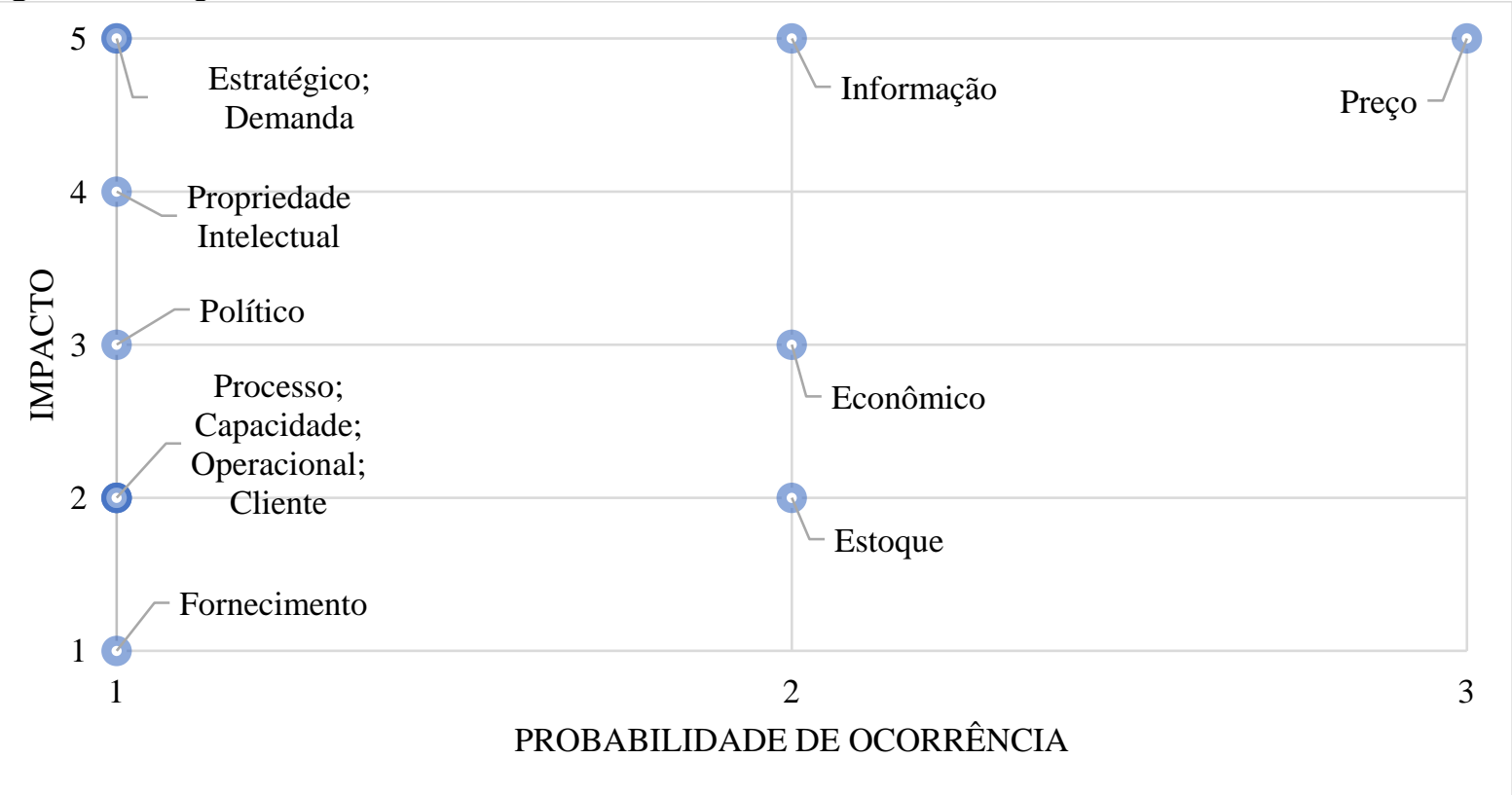

Fonte: Elaboração própria (2018)

De acordo com o diagrama, pode-se perceber que o risco de preço é prioritário, uma vez que tem alta probabilidade de ocorrência e seu impacto é severo para a organização. Tal risco ocorre devido às variações existentes nos preços das matériasprimas e no preço do produto final ao cliente. Ressalta-se que a variação de preço do gás natural praticada pelo fornecedor da empresa impacta também no preço que é repassado aos clientes, isto é, o risco é repassado para os outros membros da cadeia de suprimentos. Sendo assim, é imprescindível que os gestores estabeleçam estratégias de mitigação para esse risco, uma vez que este pode causar sérios danos à organização.

Os riscos de informação, demanda e estratégico também são críticos porque podem causar um impacto severo na organização. Entretanto, os riscos de demanda e estratégico possuem uma probabilidade baixa de ocorrência, mas podem ter um impacto bastante significativo.

A ocorrência do risco de informação está associada à indisponibilidade no sistema de informação, que é fornecido por uma empresa localizada no Estado de Pernambuco, e nos atrasos nas informações. Os problemas existentes nesse sistema são: a falta de flexibilidade e a sua interface, que torna seu uso complexo para alguns funcionários. Além disso, o sistema precisa funcionar durante todos os dias da semana, no entanto, em casos de falta de energia, o sistema fica totalmente 
indisponível, gerando atrasos no funcionamento da organização, uma vez que esta não possui gerador.

Já o risco de demanda é caracterizado pela redução no consumo de gás natural pelos clientes, principalmente, durante a crise econômica. Além disso, a empresa também enfrenta problemas devido à má previsão de demanda, pois se o cliente não consome aquele determinado volume de gás natural conforme firmado em contrato rígido, a empresa passa a sofrer penalidades de sua fornecedora de gás pelo consumo abaixo do esperado. A organização também enfrenta os riscos estratégicos devido às mudanças de preços praticadas pelos concorrentes.

Os riscos de estoque e econômico possuem uma probabilidade de ocorrência média e devem ter atenção especial dos gestores. É importante ressaltar que a empresa foi afetada diretamente pelas mudanças que ocorreram na conjuntura econômica tanto do país como do mundo, caracterizando assim o risco econômico/financeiro. Quanto ao risco de estoque, a empresa possui itens obsoletos devido às mudanças tecnológicas constantes do setor. Além disso, alguns processos que antes eram feitos internamente passaram a ser efetuados por terceiros e, dessa forma, os materiais utilizados nesse processo permaneceram em estoque.

Por sua vez, os riscos político e de propriedade intelectual também podem causar um impacto considerável na organização estudada. O risco político manifestase devido às mudanças realizadas nas regulações pertinentes ao gás natural efetuada pelos políticos. E quanto ao risco de propriedade intelectual, sua existência justificase uma vez que a empresa reduziu consideravelmente o investimento destinado à pesquisa e desenvolvimento por causa da escassez de recursos financeiros.

Os riscos de fornecimento, cliente, operacional, capacidade e processo possuem uma probabilidade de ocorrência baixa e ocasionam um impacto baixo ou nenhum para a organização. Sendo assim, esses riscos são menos críticos e não exigem nenhuma ação imediata.

Diante do exposto, verificou-se que os seguintes riscos são os mais críticos: preço, informação, estratégico e demanda. A ocorrência desses riscos pode afetar diretamente a continuidade dos negócios da empresa e da cadeia de suprimentos como um todo, gerando, consequentemente, prejuízos financeiros.

O Quadro 8 apresenta as ações de mitigação para os riscos críticos. O objetivo é reduzir tanto a frequência de ocorrência desses riscos como sua severidade. 
Quadro 8 - Ações de mitigação para os riscos críticos

\begin{tabular}{|c|l|}
\hline Tipo de Risco & \multicolumn{1}{c|}{ Ação de Mitigação } \\
\hline Preço & $\begin{array}{l}\text { - Realizar negociações com a empresa fornecedora de gás natural no que diz } \\
\text { respeito ao preço praticado por esse produto; } \\
\text { - Realizar reuniões periódicas para acompanhar as variações nos preços. }\end{array}$ \\
\hline Informação & $\begin{array}{l}\text { - Realizar pesquisas para identificar o sistema de informação adequado para as } \\
\text { necessidades da organização; } \\
\text { - Realizar treinamentos com os funcionários sobre o sistema de informação; }\end{array}$ \\
\hline Estratégico & $\begin{array}{l}\text { - Acompanhar os preços estabelecidos pelos concorrentes com a finalidade de } \\
\text { manter os preços competitivos de acordo com cada segmento atendido; }\end{array}$ \\
\hline \multirow{2}{*}{$\begin{array}{l}\text { - Adotar novos métodos estatísticos para mensurar a demanda de forma mais } \\
\text { acurada; }\end{array}$} \\
$\begin{array}{l}\text { - Manter contato constante com os clientes para acompanhar sua demanda, } \\
\text { evitando assim erros; } \\
\text { - Estimular a propaganda sobre os benefícios do uso do natural para maximizar } \\
\text { a demanda. }\end{array}$ \\
\hline
\end{tabular}

Fonte: Elaboração própria (2018)

Verifica-se que a avaliação dos riscos para a empresa investigada permitiu identificar os riscos existentes, direcionando a organização para o tratamento mais adequado a ser tomado em função de suas características. Dessa forma, a partir da ação a ser tomada, deve-se definir qual a área da empresa que deve ser mobilizada, determinando também os recursos necessários. Além disso, torna-se essencial definir responsáveis por cada ação e indicadores que devem ser monitorados.

Ressalta-se que os riscos que não foram considerados como prioritários não devem ser ignorados, uma vez que novos riscos surgem ao longo do tempo e os riscos existentes podem evoluir também.

\section{CONCLUSÕES}

De acordo com os resultados obtidos, observa-se que a organização está submetida aos riscos internos e externos à cadeia. É importante levar em consideração tanto a dimensão probabilidade de ocorrência como a dimensão impacto para avaliar os riscos.

Uma contribuição teórica foi utilizar uma vasta classificação de riscos capaz de abranger as organizações de forma sistêmica, visto que estudos anteriores utilizaram poucos tipos de riscos, como aqueles feitos por Gaudenzi e Borghesi (2006), Wu, Blackhurst e Chidambaram (2006), Schoenherr, Tummala e Harrison (2008), Funo et al. (2011) e Radivojević e Gajović (2014). 
O principal resultado prático foi apontar quais são os riscos prioritários para a organização envolvida na pesquisa. Observou-se que determinados riscos possuem a capacidade de gerar um impacto bastante alto na organização, ocasionando perdas financeiras e de reputação. Como exemplo, pode-se citar o risco de preço, que possui alta probabilidade de ocorrência e um impacto severo para a empresa. Tal risco ocorre devido às variações no preço das matérias-primas e às variações no preço do produto final ao cliente.

No entanto, ressalta-se que a organização não deve ignorar os riscos que não foram considerados como críticos, uma vez que os riscos podem evoluir e prejudicar a continuidade dos negócios. Como exemplo, pode-se citar o risco de fornecimento. Tal risco não é considerado crítico porque não impacta de forma alguma nos negócios da empresa. No entanto, as falhas nos suprimentos de materiais de consumo podem se agravar ao longo do tempo e impactar nos negócios da empresa.

Nesse contexto, recomenda-se que as estratégias de mitigação apresentadas para os riscos críticos sejam implementadas com o objetivo de reduzir a severidade e a frequência de ocorrência dos riscos identificados. Além disso, recomenda-se que a empresa também estabeleça medidas de controle para os riscos não críticos.

De forma geral, mesmo com os avanços das pesquisas em gestão de riscos em cadeias de suprimentos, quando se trata da abordagem prática, muitas empresas ainda não registram dados históricos que possam auxiliar na etapa de avaliação dos riscos. Sendo assim, sugere-se que as empresas envolvidas nesta pesquisa passem a registrar dados sobre os riscos que as acometem, uma vez que, dessa forma, seria possível estimar uma probabilidade de ocorrência do risco mais acurada.

Para as pesquisas futuras, recomenda-se que outras ferramentas, além do diagrama de risco, sejam utilizadas para realizar a avaliação do risco. Quanto ao instrumento de pesquisa utilizado, sugere-se que novos fatores de riscos sejam identificados e incorporados nos questionários para abranger melhor a realidade da organização investigada.

Por fim, apesar das limitações encontradas, verifica-se que a avaliação dos riscos realizada em uma empresa distribuidora de gás natural pode ser entendida como um primeiro passo para a gestão de riscos em uma cadeia de suprimentos como um todo. 


\section{REFERÊNCIAS}

ADHITYA, A.; SRINIVASAN, R.; KARIMI, I. A model-based rescheduling framework for managing abnormal supply chain events. Computers \& Chemical Engineering, v. 31, n. 5, p. 496-518, 2007. https://doi.org/10.1016/j.compchemeng.2006.07.002

AHMED, A.; KAYIS, B.; AMORNSAWADWATANA, S. A review of techniques for risk management in projects. Benchmarking: An International Journal, v. 14, n. 1, p. 22-36, 2007.

AQLAN, F. A software application for rapid risk assessment in integrated supply chains. Expert Systems with Applications, v. 43, p. 109-116, 2016.

https://doi.org/10.1016/j.eswa.2015.08.028

AQLAN, F; LAM, S. S. Supply chain risk modelling and mitigation. International Journal of Production Research, v. 53, n. 18, p. 5640-5656, 2015.

https://doi.org/10.1080/00207543.2015.1047975

BLACKHURST, J.; SCHEIBE, K. P.; JOHNSON, D. J. Supplier risk assessment and monitoring for the automotive industry. International Journal of Physical Distribution \& Logistics Management, v. 38, n. 2, p. 143-165, 2008.

https://doi.org/10.1108/09600030810861215

BLOME, C.; SCHOENHERR, T. Supply chain risk management in financial crises - A multiple case-study approach. International Journal of Production Economics, v. 134, n. 1, p. 43-57, 2011. https://doi.org/10.1016/j.ijpe.2011.01.002

CERYNO, P; SCAVARDA, L.; KLINGEBIEL, K. Supply chain risk: empirical research in the automotive industry. Journal of Risk Research, v. 18, n. 9, p. 1145 - 1164, 2014. https://doi.org/10.1080/13669877.2014.913662

COLICCHIA, C.; STROZZI, F. Supply chain risk management: a new methodology for a systematic literature review. Supply Chain Management: An International Journal, v. 17, n. 4, p. $403-418,2012$. https://doi.org/10.1108/13598541211246558

COLICCHIA, C; DALLARI, F.; MELACINI, M. Increasing supply chain resilience in a global sourcing context. Production Planning \& Control, v. 21, n. 7, p. 680-694, 2010.

https://doi.org/10.1080/09537280903551969

FAZLI, S.; MAVI, R.; VOSOOGHIDIZAJI, M. Crude oil supply chain risk management with DEMATEL-ANP. Operational Research, v. 15, n. 3, p. 453-480, 2015. https://doi.org/10.1007/s12351-015-0182-0

FUNO, K. A. et al. Supply chain risk management: an exploratory research in Brazilian aerospace industry. International Journal of Value Chain Management, v. 5, n. 3-4, p. 265-280, 2011. https://doi.org/10.1504/IJVCM.2011.043230

GAONKAR, R.; VISWANADHAM, N. Analytical framework for the management of risk in supply chains. Automation Science and Engineering, IEEE Transactions on, v. 4, n. 2, p. 265-273, 2007.

GARVEY, M. D.; CARNOVALE, S.; YENIYURT, S. An analytical framework for supply network risk propagation: A Bayesian network approach. European Journal of Operational Research, v. 243, n. 2, p. 618-627, 2015. https://doi.org/10.1016/j.ejor.2014.10.034 
GAUDENZI, B.; BORGHESI, A. Managing risks in the supply chain using the AHP method. The International Journal of Logistics Management, v. 17, n. 1, p. 114-136, 2006. https://doi.org/10.1108/09574090610663464

GHADGE, A.; DANI, S.; KALAWSKY, R. Supply chain risk management: present and future scope. The International Journal of Logistics Management, v. 23, n. 3, p. 313-339, 2012. https://doi.org/10.1108/09574091211289200

HALLIKAS, J. et al. Risk management processes in supplier networks. International Journal of Production Economics, v. 90, n. 1, p. 47-58, 2004.

https://doi.org/10.1016/j.ijpe.2004.02.007

HARLAND, C.; BRENCHLEY, R.; WALKER, H. Risk in supply networks. Journal of Purchasing and Supply management, v. 9, n. 2, p. 51-62, 2003.

https://doi.org/10.1016/S1478-4092(03)00004-9

HECKMANN, I.; COMES, T.; NICKEL, S. A critical review on supply chain risk -Definition, measure and modeling. Omega, v. 52, p. 119-132, 2015.

https://doi.org/10.1016/j.omega.2014.10.004

HO, W. et al. Supply chain risk management: a literature review. International Journal of Production Research, v. 53, n. 16, p. 5031-5069, 2015.

https://doi.org/10.1080/00207543.2015.1030467

KERN, D. et al. Supply risk management: model development and empirical analysis.

International Journal of Physical Distribution \& Logistics Management, v. 42, n. 1, p. 60 - 82, 2012. https://doi.org/10.1108/09600031211202472

LACERDA, R.; ENSSLIN, L.; ENSSLIN, S. Uma análise bibliométrica da literatura sobre estratégia e avaliação de desempenho. Gestão \& Produção, v. 19, n. 1, p. 59-78, 2012. https://doi.org/10.1590/S0104-530X2012000100005

LAVASTRE, O.; GUNASEKARAN, A.; SPALANZANI, A. Effect of firm characteristics, supplier relationships and techniques used on Supply Chain Risk Management (SCRM): an empirical investigation on French industrial firms. International Journal of Production Research, v. 52, n. 11, p. 3381-3403, 2014. https://doi.org/10.1080/00207543.2013.878057

MANUJ, I.; MENTZER, J. Global supply chain risk management strategies. International Journal of Physical Distribuition \& Logistics Management, v. 38, n. 3, p. $192-223$, 2008. https://doi.org/10.1108/09600030810866986

MANUJ, I.; MENTZER, J.; BOWERS, M. Improving the rigor of discrete-event simulation in logistics and supply chain research. International Journal of Physical Distribution \& Logistics Management, v. 39, n. 3, p. 172-201, 2009. https://doi.org/10.1108/09600030910951692

MATOOK,S. LASCH, R. e TAMASCHKE,R. Supplier development with benchmarking as part of a comprehensive supplier risk management framework. International Journal of Operations \& Production Management, v. 29, n. 3, p. 241-267, 2009.

https://doi.org/10.1108/01443570910938989

NORRMAN, A.; JANSSON, U. Ericsson's proactive supply chain risk management approach after a serious sub-supplier accident. International Journal of Physical Distribution 
\&Logistics Management, v. 34, n. 5, p. 434-456, 2004. https://doi.org/10.1108/09600030410545463

PUNNIYAMOORTHY, M.; THAMARAISELVAN, N.; MANIKANDAN, L. Assessment of supply chain risk: scale development and validation. Benchmarking: An International Journal, v. 20, n. 1, p. 79 - 105, 2013. https://doi.org/10.1108/14635771311299506

QAZI, A., QUIGLEY, J.; DICKSON, A. Supply Chain Risk Management: Systematic literature review and a conceptual framework for capturing interdependencies between risks.

Proceedings of the $\mathbf{2 0 1 5}$ International Conference on Industrial Engineering and Operations Management, Dubai, United Arab Emirates, p. 1 -13, 2015.

RADIVOJEVIĆ, G.; GAJOVIĆ, V. Supply chain risk modeling by AHP and Fuzzy AHP methods. Journal of Risk Research, v. 17, n. 3, p. 337-352, 2014.

https://doi.org/10.1080/13669877.2013.808689

RAO, S.; GOLDSBY, T. Supply chain risks: a review and typology. The International Journal of Logistics Management, v. 20, n. 1, p. 97-123, 2009.

https://doi.org/10.1108/09574090910954864

SCHOENHERR, T.; TUMMALA, V. M. R.; HARRISON, T. Assessing supply chain risks with the analytic hierarchy process: Providing decision support for the offshoring decision by a US manufacturing company. Journal of Purchasing and Supply Management, v. 14, n. 2, p. 100-111, 2008. https://doi.org/10.1016/i.pursup.2008.01.008

SHI, D. A review of enterprise supply chain risk management. Journal of Systems Science and Systems Engineering, v. 13, n. 2, p. 219-244, 2004. https://doi.org/10.1007/s11518-006$\underline{0162-2}$

SINGHAL, P.; AGARWAL, G.; MITTAL, M. Supply chain risk management: review, classification and future research directions. International Journal of Business Science and Applied Management, v. 6, n. 3, p. $15-42,2011$.

SINHA, P. R.; WHITMAN, L. E.; MALZAHN, D. Methodology to mitigate supplier risk in an aerospace supply chain. Supply Chain Management: An International Journal, v. 9, n. 2, p. 154-168, 2004. https://doi.org/10.1108/13598540410527051

SODHI, M.; SON, B.; TANG, C. Researchers' Perspectives on Supply Chain Risk Management. Production and Operations Management, v. 21, n. 1, p. 1- 13, 2012. https://doi.org/10.1111/j.1937-5956.2011.01251.x

TANG, C.; TOMLIN, B. The power of flexibility for mitigating supply chain risks. International Journal of Production Economics, v. 116, n. 1, p. 12-27, 2008.

https://doi.org/10.1016/j.ijpe.2008.07.008

TANG, O.; MUSA, S. N. Identifying Risk Issues and Research Advancements in Supply

Chain Risk Management. International Journal of Production Economics, v. 133, n. 1, p. 25 - 34, 2011. https://doi.org/10.1016/j.ijpe.2010.06.013

THUN, J.; HOENIG, D. An empirical analysis of supply chain risk management in the German automotive industry. International Journal of Production Economics, v. 131, n. 1, p. 242-249, 2011. https://doi.org/10.1016/j.ijpe.2009.10.010 
TRKMAN, P.; MCCORMACK, K. Supply chain risk in turbulent environments - A conceptual model for managing supply chain network risk. International Journal of Production

Economics, v. 119, n. 2, p. 247-258, 2009. https://doi.org/10.1016/j.ijpe.2009.03.002

TUMMALA, R.; SCHOENHERR, T. Assessing and managing risks using the supply chain risk management process (SCRMP). Supply Chain Management: An International

Journal, v. 16, n. 6, p. $474-483,2011$. https://doi.org/10.1108/13598541111171165

TUNCEL, G.; ALPAN, G. Risk assessment and management for supply chain networks: A case study. Computers in Industry, v. 61, n. 3, p. 250-259, 2010.

https://doi.org/10.1016/j.compind.2009.09.008

VILKO, J. P. P.; HALLIKAS, J. M. Risk assessment in multimodal supply

chains. International Journal of Production Economics, v. 140, n. 2, p. 586-595, 2012. https://doi.org/10.1016/.i.jpe.2011.09.010

WAGNER, S. M.; BODE, C. Dominant risk and Risk management practices in supply chains. In: ZSIDISIN, G. A.; RITCHIE, B. (Eds.). Supply Chain Risk: A Handbook of Assessment, Management, and performance. USA: Springer, 2009. p. 271-290.

https://doi.org/10.1007/978-0-387-79934-6 17

WATERS, D. Supply Chain Risk Management: Vulnerability and Resilience in Logistics. Philadelphia: Kogan Page, 2007.

WU, T.; BLACKHURST, J.; CHIDAMBARAM, V. A model for inbound supply risk analysis. Computers in industry, v. 57, n. 4, p. 350-365, 2006.

https://doi.org/10.1016/j.compind.2005.11.001

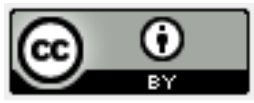

Artigo recebido em: 30/01/2018 e aceito para publicação em: 29/07/2018

DOI: http://dx.doi.org/10.14488/1676-1901.v18i3.3131 